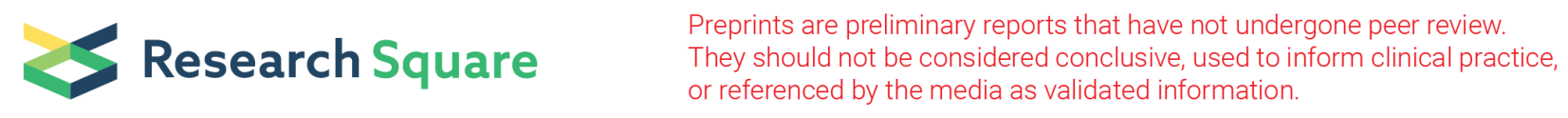

\title{
Dasabuvir Suppresses Esophageal Squamous Cell Carcinoma Growth As a Novel ROCK1 Inhibitor
}

\author{
Xinning Liu \\ Zhengzhou University \\ Yanan Jiang \\ Zhengzhou University \\ Hao Zhou \\ Zhengzhou University \\ Mingzhu Li \\ Zhengzhou University \\ Zhuo Bao \\ Zhengzhou University \\ Zitong Wang \\ Zhengzhou University \\ Chenyang Zhang \\ Zhengzhou University \\ Zhenliang Xie \\ Zhengzhou University \\ Jimin Zhao \\ Zhengzhou University \\ Zigang Dong \\ Zhengzhou University \\ Zhiping Guo \\ Zhengzhou University \\ Kangdong Liu ( $\nabla$ kdliu@zzu.edu.cn ) \\ Zhengzhou University
}

Research

Keywords: Esophageal squamous cell carcinoma, ROCK1, MAPK1, CDK4, cyclin D1

Posted Date: July 19th, 2021

DOl: https://doi.org/10.21203/rs.3.rs-709730/v1

License: (1) This work is licensed under a Creative Commons Attribution 4.0 International License. Read Full License 


\section{Abstract}

Background: Esophageal squamous cell carcinoma (ESCC) is a high recurrence rate of upper-digestive cancer with a low 5-year survival rate. Therefore, there is an urgent need for effective chemopreventive drugs that can extend the survival rate of patients. Through screening of FDA-approved drugs, dasabuvir was found to suppress ESCC proliferation.

Methods: Cell number count assay was used to screen for drugs with inhibitory effect on ESCC cells and detect the inhibitory effect of dasabuvir on proliferation of ESCC cells KYSE150 and KYE450. Phosphoproteomics and proteomics were used to investigate the mechanism of dasabuvir inhibiting ESCC. In vitro kinase assay was used to verify the inhibition of extracellular signal-regulated kinase 1/2 (ERK1/2) activation by ROCK1 by dasabuvir. The PDX model was used to test the inhibitory effect of dasabuvir on ESCC in vivo.

Results: In this study, we found that dasabuvir is a novel inhibitor of Rho-associated protein kinase 1 (ROCK1). Dasabuvir inhibited the growth of the KYSE150 and KYSE450 ESCC cell lines in a time and dose-dependent manner and arrested cell cycle at the G0/G1 phase. The antitumor activity was validated in vivo using a patient-derived xenograft tumor model in mice. Dasabuvir inhibited the activation of ERK1/2 by ROCK1 and downregulated cyclin-dependent kinase 4 (CDK4) and cyclin D1 expression.

Conclusions: These results provide the first evidence that dasabuvir serves as a ROCK1 inhibitor, suppresses ESCC growth in vivo and in vitro, and arrests the cell cycle through the ROCK1/ERK signaling pathway.

\section{Background}

Esophageal cancer is a gastrointestinal tumor ranks 7 th in terms of incidence $(604,000$ new cases) and 6 th in overall mortality (544,000 deaths) worldwide[1]. In addition, $90 \%$ of esophageal cancer cases are esophageal squamous cell carcinoma (ESCC)[2]. At present, surgery, chemotherapy, radiotherapy, and limited targeted therapy have poor prognoses for esophageal cancer[3, 4]. Therefore, there is an urgent need to find effective chemopreventive drugs for ESCC. Screening drugs approved by the FDA is an effective strategy for finding drugs to prevent the occurrence and recurrence of cancer. Investigating FDA-approved drugs with safety data and detailed pharmacokinetics can reduce development cycles, save development costs and make full use of resource[6, 7].

Through screening FDA-approved drugs, we found dasabuvir(Fig. 1a), an anti-hepatitis C virus drug, had an obvious inhibitory effect on ESCC cells. Dasabuvir can combine with other direct-acting antiviral drugs to achieve high cure rates in a variety of interferon-free regiments and low adverse reactions as a nonnucleoside NS5B polymerase inhibitor[8-10].

ROCK1 (Rho-associated coiled-coil kinases 1) is a classical serine-threonine kinase which could regulate the cytoskeleton through phosphorylating the downstream substrates and increasing the stability of actin filament and generation of actomyosin contractility[11]. ROCK1 plays an important role in regulating cell movement, angiogenesis, and migration, and can promote the growth, proliferation, and survival of tumor cells by regulating the tumor microenvironment[12]. ROCK1 has been found to be associated with dozens of cancers such as prostate cancer[13-16], laryngeal squamous cell carcinomas[17], nasopharyngeal carcinoma[18], osteosarcoma[19, 20], breast cancer[21], and gastric cancer[22]. It was reported that the RhoA/ROCK1 pathway was associated with the EMT process of ESCC, lymph node metastasis, and clinical staging of ESCC. Overexpression of ROCK1 was also significantly associated with the progression of ESCC and predicted poor prognosis[23-25]. These data indicate that ROCK1 is a potential target for ESCC treatment or chemoprevention.

In this study, we found dasabuvir had an obvious cytotoxic effect on ESCC cells, and effectively inhibited the proliferation and anchor-independent and dependent growth of ESCC cells in vitro. Dasabuvir is a novel ROCK1 inhibitor that can block the ROCK1/ERK signaling pathway and downregulate the expression of CDK4 and cyclin D1 thus arresting ESCC cells in the G0/G1 phase. Importantly, dasabuvir can inhibit the growth of ESCC tumors in vivo. This provides an experimental basis for future clinical application of dasabuvir for ESCC chemoprevention.

\section{Material And Methods}

\section{Chemicals}

Dasabuvir (CAS:1132935-63-7) was purchased from ATK Chemical Company (Shanghai, China); dasabuvir Sodium Tablets (Exviera) used in vivo was purchased from AbbVie Ireland NL B.V (Sligo, Ireland). DAPI, PBS, RNase and PI were purchased from Solarbio Science \& Technology Co (Beijing, China). Polybrene, DMSO and BME powder were purchased from Sigma-Aldrich (Shanghai) Trading Co, Ltd (Shanghai, China).

\section{Cell culture}

The ESCC cell lines (KYSE150 and KYSE450) were purchased from the Chinese Academy of Sciences cell bank (Shanghai, China). These cell lines were authenticated by STR profiling and cultured as described previously[26].

\section{Cell toxicity and proliferation assay}

Cell viability and proliferation assays were performed as described previously[26]. The cell number was assessed by DAPI staining after treatment with dasabuvir $(0,2.5,5,10$, or $15 \mu \mathrm{M})$ for $0,24,48,72$, or $96 \mathrm{~h}$. The cells were photographed and counted using IN Cell Analyzer 6000 (GE Healthcare, American U.S.) and the knockdown cells were counted by MTT assay. 
The anchorage-independent cell growth assays were performed as described previously[24]. 8000 cells were seeded into each well of a 6-well plate. After culturing for 7-14 days, the cell clones were photographed and counted using IN Cell Analyzer 6000.

\section{Anchorage-dependent cell growth assay}

Each well of 6-well plate was seeded with 200 cells. After culturing with different concentrations of dasabuvir $(0,2.5,5,10$, or $15 \mu \mathrm{M})$ for $7-10$ days, the cell clones were fixed, stained and counted.

\section{Mass Spectrometry and omics analysis}

Treating KYSE150 cells with $15 \mu \mathrm{M}$ dasabuvir for $24 \mathrm{~h}$. The cells were then collected and lysed by ultrasonic wave. After centrifugation, the protein concentration was determined. Trypsin enzymatic hydrolysis was performed to obtain polypeptides. After modification and enrichment, the polypeptides were separated and analyzed by mass spectrometry. Maxquant(v1.5.2.8) was used to retrieve secondary mass spectrometry data.

\section{Protein extracts and Westem blotting analysis}

KYSE150 and KYSE450 cells were treated with dasabuvir $(0,2.5,5,10$, or $15 \mu \mathrm{M})$ for $24 \mathrm{~h}$. Protein extracts and Western blotting analysis were performed as described previously[27]. The primary antibodies made against ROCK1 (CST: 4035T), phospho-ERK1/ERK2 (Thr185, Tyr187; Invitrogen: 700012), P44/42 MAPK (ERK1/2; CST: 9102S), Cyclin D1 (Wanlei: WL01435a), and CDK4 (CST: 12790 ) were used at 1:1000 dilutions. The secondary antibody signals were detected using a chemiluminescence reagent.

\section{Kinase prediction, target prediction, and correlation analysis}

The kinase prediction of dasabuvir was carried out using iGPS1.0 (http://igps.biocuckoo.org/). The target prediction for dasabuvir was performed using SwissTargetPrediction (http://www.swisstargetprediction.ch/). Correlation analysis of ROCK1 and MAPK1 was performed using the TCGA database (https://www.aclbi.com/static/index.html\#/).

\section{Computational modeling of dasabuvir with ROCK1}

The docking of Dasabuvir to ROCK1 was performed using the Schrodinger Suite 2016 software program, and the ROCK1 crystal structure was downloaded from the PDB Bank (PDB: 2ESM).

\section{Ex vivo and in vitro pull-down assays}

Preparation of dasabuvir-Sepharose 4B beads was performed as reported[28]. KYSE150, KYSE450, 293T and $293 \mathrm{~F}$ cell lysates (500 $\mu \mathrm{g}$ ), recombinant human active ROCK1 $(200 \mathrm{ng})$ were incubated with dasabuvir-Sepharose $4 \mathrm{~B}(100 \mu \mathrm{L})$ and Sepharose $4 \mathrm{~B}(100 \mu \mathrm{L})$ alone (as a control) beads in reaction buffer. The proteins bound were verified through Western blotting.

\section{ATP competition assay}

Recombinant human active ROCK1 (100 ng) were incubated with dasabuvir-Sepharose $4 \mathrm{~B}(100 \mu \mathrm{L})$ and Sepharose $4 \mathrm{~B}(100 \mu \mathrm{L})$ alone (as a control) beads in reaction buffer with different concentration $\mathrm{ATP}(10$ or $100 \mu \mathrm{M})$. The proteins bound were verified through Western blotting.

\section{In vitro kinase assay}

The kinase reaction system was consisted of recombinant human active ROCK1 (30 ng), ERK1 protein (300 ng), ERK2 protein (150 ng), ATP (20 $\mu$ M) and kinase buffer $(25 \mu \mathrm{L})$. The total reaction system was placed in a $30^{\circ} \mathrm{C}$ water bath for 30 min incubation. Then ROCK 1 kinase activity was verified by Western blotting.

\section{Protein purification}

ROCK1 (NM_005406) cDNA clone (number: G124885) was purchased from YouBia Biotechnology Company (Chongqing, China). Rock1 kinase domian $₫ 117-$ 535aa『and the mutated ROCK1 kinase domain (M156A, L202A, and D205A) PCR product were inserted into pGEX-6p-1 vector between Smal and Sall restriction sites to obtain pGEX-6p-1-ROCK1 and ROCK1 (M156A, L202A, and D205A) plasmids(Table 1). These plasmids were transformed into chemically competent E. coli BL21 (DE3) cells. The harvested cells were lysed via sonication and centrifuged. The recombinant ROCK1 protein was purified through a HisTrap column (GE Healthcare) and a HiTrap Q column (GE Healthcare), and then loaded onto a Superdex 200 10/300 gel filtration column.

Table 1 Primer sequences for mutated ROCK1 for RT-qPCR designed by SnapGene. 


\begin{tabular}{|lll|}
\hline Gene & Forward05'-3'0 & Forward05'-3'0 \\
\hline ROCK1 & TCTACATGGTGATGGAATACGCCCCTGGTGGAGATCTTGTAAA & TTTACAAGATCTCCACCAGGGGCGTATTCCATCACCATGTAGA \\
(Met156) & & \\
\hline ROCK1 & TTCACAGAGATGTGAAGCCTGCCAACATGCTGCTGGATAAATC & GATTTATCCAGCAGCATGTTGGCAGGCTTCACATCTCTGTGAA \\
(Leu202) & & \\
\hline ROCK1 & ATGTGAAGCCTGATAACATGGCCCTGGATAAATCTGGACATTT & AAATGTCCAGATTTATCCAGGGCCATGTTATCAGGCTTCACAT \\
(Asp205) & & \\
\hline
\end{tabular}

Immunofluorescence assay

Cells were incubated overnight at $4{ }^{\circ} \mathrm{C}$ with primary antibodies containing ROCK1 (SCBT: sc-17794) and p-MAPK1, followed by secondary antibodies containing FITC (Abbkine: A22120) and TRTIC (GeneTex: GTX26744) for 2 h. After DAPI staining, the images were captured by IN Cell Analyzer 6000 and analyzed by Image J.

\section{Gene Set Enrichment Analysis}

GSEA V4.1.0 software package was used to analyze the differences in protein expression between the treatment group and the control group in phosphoproteomics and proteomics data.

\section{Cell cycle assay}

Cells were plated into $60-\mathrm{mm}$ culture dishes $\left(2 \times 10^{5} \mathrm{cells} / \mathrm{dish}\right)$. The cells were starved for $24 \mathrm{~h}$ and treated dasabuvir. Cells were fixed in $1 \mathrm{~mL}$ of cold $70 \%$ ethanol and stored at $-20{ }^{\circ} \mathrm{C}$ for $24 \mathrm{~h}$. After treated with RNase $(100 \mathrm{mg} / \mathrm{mL})$ and stained with $\mathrm{Pl}(20 \mathrm{mg} / \mathrm{mL})$. Cells were then analyzed by Flow Cytometer $(\mathrm{BD}$ Biosciences, San Jose, CA).

\section{Generation of stable ROCK1 knock-down cell lines}

The ROCK1 shRNA sequences (Table 2) were designed using the siRNAext program (http://jura.wi.mit.edu siRNAext). These shROCK1 plasmids were transferred to 293T cells to collect the shRNA lentiviral particles. KYSE150 and KYSE450 cells ( $60 \%$ confluent) were cultured with DMEM containing $8 \mu \mathrm{g} / \mathrm{mL}$ polybrene and shRNA lentiviral particles. A medium containing puromycin (KYSE150 $2 \mu \mathrm{g} / \mathrm{mL}, \mathrm{KYSE} 4501 \mu \mathrm{g} / \mathrm{mL}$ ) was used to select for shROCK1 cells.

Table 2 The shRNA sequences for ROCK1

\begin{tabular}{|c|c|c|}
\hline shROCK1 & Forward 05 '-3' & Forward 05 '-3' \\
\hline$\# 1$ & CCGGGCCAGCAAAGAGAGTGATATTCTCGAGAATATCACTCTCTTTGCTGGCTTTTTG & AATTCAAAAAGCCAGCAAAGAGAGTGATATTCTCGAGAA' \\
\hline \#2 & CCGGGTGGAGATCTTGTAAACTTAACTCGAGTTAAGTTTACAAGATCTCCACTTTTTG & AATTCAAAAAGTGGAGATCTTGTAAACTTAACTCGAGTT/ \\
\hline \#3 & CCGGGCATTCCAAGATGATCGTTATCTCGAGATAACGATCATCTTGGAATGCTTTTTG & AATTCAAAAAGCATTCCAAGATGATCGTTATCTCGAGATA \\
\hline \#4 & CCGGCGGTTAGAACAAGAGGTAAATCTCGAGATTTACCTCTTGTTCTAACCGTTTTTG & AATTCAAAAACGGTTAGAACAAGAGGTAAATCTCGAGAT' \\
\hline \#5 & CCGGGCACCAGTTGTACCCGATTTACTCGAGTAAATCGGGTACAACTGGTGCTTTTTG & AATTCAAAAAGCACCAGTTGTACCCGATTTACTCGAGTA\& \\
\hline
\end{tabular}

\section{Patient-derived xenograft mouse model}

Female SCID mice (5-6 weeks) were purchased from Vital River (Beijing, China). The study protocol was approved by the Animal Care and Use Committee of Zhengzhou University (Zhengzhou, Henan Province, China). The samples of ESCC tumor tissue were obtained from the Linzhou Tumor Hospital. All patients provided written informed consent to use the tissue samples. EG20 was from a male, 46 years old, with T2NOMOII, moderately differentiated. LEG110 was from a male, 69 years old, T3N1M0IIlb, with moderately differentiated medullary squamous cell carcinoma. LEG34 was from a female, 68 years old, T4NOMOIII, with moderately differentiated medullary squamous cell carcinoma. The process of building the PDX mouse model has been described previously[29]. When the average tumor volume reached $100 \mathrm{~mm}^{3}$, mice were divided randomly into 3 groups: gavage with solvent control ( $0.9 \%$ saline), lowdose dasabuvir (10 mg/kg) or high-dose dasabuvir $(50 \mathrm{mg} / \mathrm{kg})$ made using dasabuvir sodium tablets until the average tumor volume of the control group reached $1000 \mathrm{~mm}^{3}$. The weight of the mice was monitored every 2 days, and tumor volume was measured every 3 days. Tumor volume was calculated using the following formula: tumor volume $=$ length $\times$ width ${ }^{2} / 2$.

\section{Immunohistochemical}

Tumor tissue sections of $5 \mu \mathrm{m}$ paraffin were prepared, dewaxed, exposed to antigen, and incubated overnight with primary antibody (1:50) at $4{ }^{\circ} \mathrm{C}$. The secondary antibody was incubated at $37^{\circ} \mathrm{C}$ for $30 \mathrm{~min}$, then stained with DAB and restained with hematoxylin. After dehydration, slides were installed, scanned by tissue fax (version 4.2) and analyzed by the Image Pro Plus software program (Media Cybernetics, Rockville, MD).

\section{Statistical analysis}


On the premise of homogeneity of variance, the difference between the two groups was calculated by a one-way ANOVA test (SPSS 20.0). All experiments were performed at least three times, and $p<0.05$ was considered significant.

\section{Results}

\section{Dasabuvir inhibits ESCC cell proliferation in vitro}

Dasabuvir (CAS \#1132935-63-7, Fig. 1b) showed obvious cytotoxicity to KYSE450 cells in vitro in screening of FDA-approved drugs in a cytotoxicity assay at a concentration of $50 \mu \mathrm{M}$ (Fig. 1a, Fig. S1a). To further assess its inhibitory effects, we treated the human ESCC cell lines KYSE150 and KYSE450 with various concentrations $(0,2.5,5,10$, or $15 \mu \mathrm{M})$ of dasabuvir dissolved in DMSO, according to the IC50 in these two cell lines (Fig. 1c). The results indicated that dasabuvir markedly suppressed cell growth in a dose-dependent manner (Fig. 1d). In the anchorage-independent (Fig. 1e) and anchorage-dependent (Fig. 1f) ESCC cell growth assays, dasabuvir showed strong dose-dependent inhibition of growth. These data indicated that dasabuvir effectively inhibited ESCC proliferation in vitro.

\section{Dasabuvir plays a role in the ROCK1/ERK signaling pathway}

To evaluate the molecular mechanism of dasabuvir, phosphoproteomics were analyzed after KYSE150 cells were treated with dasabuvir for 24 h. Compared with the control group (DMSO), 13834 phosphorylation sites were identified on 3481 proteins, among which 8387 sites of 2952 proteins contained quantitative information. To ensure that the results were reliable, the identification data was filtered by a localization probability $>0.75$. Finally, 8863 phosphorylation modification sites were identified on 3177 proteins, of which 7299 sites of 2815 proteins contained quantitative information. The protein quantification group was used for normalization, to remove the influence of protein expression on the modified signal, and the data would be used subsequently for bioinformatics analysis(Fig. S1b and S1c).

The different sites were screened for a 1.5 -fold change threshold and a $t$-test $P$-value $<0.05$. Based on these data and criteria, the modification levels of 238 sites in the dasabuvir treatment group were found to be upregulated, and 446 sites were downregulated (Fig. 2a and $2 b$ ). For further analysis, we enriched the phosphorylation sites with obvious downregulation (Fig. 2c), and these sites were induced into the eight pathways (Fig. 2d) for further analysis. The downregulated phosphorylation sites in these pathways were analyzed again, and MAPK1 T185 and Y187 (also known as extracellular signal-regulated kinase, ERK2) were enriched in 6 of the 8 downregulated pathways (Fig. 2e; Fig. S1d to S1f). Western blotting results also confirmed the down-regulation of pERK1/2(Fig. 2f).

To explore the mechanism of action of dasabuvir on ESCC, we performed kinase activity analysis based on mass spectrometry results. IGPS1.0 was used to predict upstream kinases based on phosphorylation sites. Based on the assumption that similar short peptides may perform similar biological functions, and that similar protein kinases may regulate similar short motifs, the software predicts kinase proteins that may regulate specific phosphorylation sites, and then uses protein-protein interaction information to filter out potential false positive interactions. There were 38284 regulatory relationships between 303 protein kinases and 3492 phosphorylation sites on 1372 proteins identified (Fig. 2g).

Since the phosphorylation expression level may reflect the regulatory state of kinases, enrichment analysis was used to further predict the activity of upstream kinases by iGPS1.0. Based on the above methods, we predicted a total of 38,284 regulatory relationships between 303 protein kinases and 3492 phosphorylation sites on 1372 proteins identified by the project. A kinase regulatory network was constructed to observe the regulatory relationship between kinases and substrates. We enriched ROCK1 and found that the kinase activity of ROCK1 was inhibited, and it could regulate five different sites. ROCK1 ranked first among the predicted targets from SwissTargetPrediction. This indicated that ROCK1 was a direct target of dasabuvir.

ROCK mediates MAPK signaling[30]. In addition, ROCK1 siRNA decreases the phosphorylation of MAPK in the cell nucleus[31, 32]. The TCGA database showed that the levels of ROCK1 and MAPK1 were positively correlated in ESCC. Consequently, dasabuvir might inhibit cell proliferation via the ROCK1/ERK signaling pathway in ESCC (Fig. 2g).

\section{Dasabuvir binds and inhibits ROCK1}

To determine whether ROCK1 is a direct target of dasabuvir, blind docking of dasabuvir to the ROCK1 protein was performed using Autodock4, by setting grid sizes that included the ROCK1 kinase domain template. The ROCK1 kinase domain, which comprises of the N-terminal region accounting for approximately one-third of the full-length sequence of ROCK1 was obtained from the PDB database. The ROCK1 kinase domain consists of an N-terminal helical domain (residues 5-72), a bilobed kinase domain (residues 73-356), and a kinase tail (residues 357-405)[33]. The docking results indicated that dasabuvir bound to ROCK1 at Met156, Leu202, and Asp205 (Fig. 3a), and that these binding sites belong to the protein kinase domain of ROCK1.

To verify this binding model, we conjugated dasabuvir with Sepharose 4B beads and conducted in vitro and ex vivo pull-down assays. The active ROCK1 kinase domain bound to Sepharose 4B beads conjugated with dasabuvir, but not to Sepharose 4B beads alone (Fig. 3b) in an in vitro pull-down assay. To confirm whether dasabuvir binds with full-length ROCK1, an ex vivo pull-down assay was performed using exogenous and endogenous ROCK1. Using 293T and 293F cells transfected with pcDNA3.1-Rock1-HA, exogenous full-length ROCK1 protein bound to dasabuvir (Fig. 3c and 3d). The results also show that dasabuvir directly binds with endogenous ROCK1 in KYSE150 and KYSE450 cells (Fig. 3e and 3f). In addition, the combination of dasabuvir and ROCK1 was obviously affected by the presence of ATP(Fig. $3 g$ ).

MEK1/2 was tested for binding with dasabuvir as a direct upstream of ERK1/2. The results indicated that MEK1/2 did not bind with dasabuvir (Fig. S2a and S2b). In order to further verify the binding sites of ROCK1 to dasabuvir, we mutated the ROCK1 kinase domain residues at Met156, Leu202, and Asp205 to alanine, and purified the wild-type recombinant ROCK1 kinase domain protein and mutated ROCK1 protein (Fig. S2c). Dasabuvir bound to the wild-type ROCK1

Page 5/16 
protein but not the mutant protein (Fig. 3h). Comparison of ROCK1 sequences from different species also indicated that the ROCK1 sequence was highly conserved at these binding sites (Fig. 3i), which may be important for the role of dasabuvir. These results suggest that dasabuvir binds to ROCK1 through the Met156, Leu202, and Asp205 sites in an ATP-competing manner.

An in vitro kinase assay was conducted to examine whether ROCK1 activity can be inhibited by dasabuvir. Activated recombinant ROCK1 kinase protein was mixed with human recombinant ERK1 and ERK2 in the presence of different concentrations of dasabuvir to assess phosphorylation of ERK1 and ERK2. The results showed that phosphorylation of ERK1 and ERK2 were markedly reduced (Fig. 3j and 3k), demonstrating that dasabuvir can significantly inhibit ROCK1 kinase activity in a dose-dependent manner. Taken together, these results indicated that dasabuvir is an ATP-competed inhibitor of ROCK1.

\section{Dasabuvir induces G0-G1 cell cycle arrest of KYSE150 and KYSE450 cells through the ROCK1/ERK signal pathway}

Immunofluorescence showed that ROCK1 and p-ERK1/2 overlapped significantly in untreated ESCC cells; dasabuvir blocked the co-localization of the two molecules, and the fluorescence overlap between them became weak (Fig. 4a). These data indicated that dasabuvir inactivates the ROCK1/ERK signaling pathway in ESCC cells. To further investigate how dasabuvir affects the progression of ESCC through the ROCK1/ERK signaling pathway, GSEA enrichment was performed on the phosphorylation and proteomics data. The GSEA results showed that dasabuvir affected the cell cycle signaling pathway of ESCC (Fig. 4b and 4c; Fig. S3a and S3b). ROCK1 can regulate the transcription and expression of G1 checkpoint proteins like cyclin D1,CDK2, and CDK4, then ROCK1 can enhance cell proliferation by promoting cell cycle transition from G0/G1 phase to $S$ phase[34]. The significantly downregulated proteomic protein sites were enriched, the corresponding pathways were located and intersected, and CDK4 was identified (Fig. 4d to 4f; Fig. S4a to S4e). Western blotting analysis indicated that dasabuvir inhibited the expression of p-ERK1/2, cyclin D1, and CDK4 in a dose-dependent manner (Fig. 4g). Cell cycle experiments showed that dasabuvir significantly blocked the cells at the G0/G1 phase (Fig. 4h), which was consistent with the downregulation of CDK4 and cyclin D1 expression.

\section{Dasabuvir exerts cancer cell inhibition through ROCK1}

Underlying the TCGA and GEPIA data, the expression of ROCK1 in esophageal cancer was much higher than that in most cancers (Fig. 5a) and the expression of ROCK1 in esophageal cancer was higher than that in normal tissue (Fig. 5b). We selected KYSE150 and KYSE450 cell lines to conduct further studies. In addition, we used virus particles containing shRNA mock or shRNA ROCK1 to infect cells to prepare ROCK1 knockdown cells. Finally, we chose sequences 1 and 2 (Fig. 5c) and conducted a proliferation assay (Fig. 5d) and an anchorage-dependent cell growth assay (Fig. 5e). Compared with mock-infected cells, knockdown of ROCK1 in ESCC cells resulted in suppression of cell proliferation and anchorage-dependent cell growth (Fig. 5d and 5e). Furthermore, downregulated ROCK1 also inhibited the expression of the ERK-CDK4/Cyclin D1 signaling pathway (Fig. 5f). p-ERK1/2, T-ERK1/2, CDK4 and cyclinD1 were obviously down-regulated with the knockdown of ROCK1, the expression of each target was reduced by at least $30 \%$, and in some cases by as much as $90 \%$ such as p-ERK1/2 compared with mock-infected cells. With the inactivation of ERK-CDK4/Cyclin D1 signaling pathway, the cell cycle of KYSE150 and KYSE450 cells was also arrested in the G0/ G1 phase (Fig. $5 \mathrm{~g}$ ). Compared with mock cells, we also found that the inhibitory effect of dasabuvir was attenuated on shROCK1-1 cells and dasabuvir lost its inhibitory effect on shROCK1-2 cells proliferation (Fig. 5h). These data also indicate that dasabuvir exerts cancer cell inhibition through ROCK1.

\section{Dasabuvir reduces ESCC PDX xenograft growth in vivo}

The PDX mouse model is one of the best models to evaluate the antitumor activity of drugs in vivo[35]. Our group has established an ESCC PDX platform to screen for drugs (Fig. 6a)[36]. In order to verify the inhibitory effect of dasabuvir in vivo, we chose three ESCC PDX models (EG20, LEG34, LEG110) to conduct further studies (Fig. $6 \mathrm{c}$ to $6 \mathrm{~d}$ ). After SCID mice were implanted with tumor fraction, physiological saline or dasabuvir (10 mg/kg and $50 \mathrm{mg} / \mathrm{kg}$ ) was administered via gavage once daily. After searching for the drug instructions, we found that the recommended dosage of dasabuvir sodium tablets was 500 $\mathrm{mg}$ per day for people. By conversion, the dose for mice was $75 \mathrm{mg} / \mathrm{kg}$, and our actual dose was much lower than the recommended dose. We also saw no significant weight change in the mice treated with the drug compared to the vehicle group (Fig. S5a).

In these three cases, dasabuvir effectively inhibited tumor growth and tumor volume compared with the vehicle group (Fig. $6 \mathrm{c}$ to $6 \mathrm{~d}$ ). In addition, the effect of dasabuvir on Ki-67 and p-ERK1/2 were evaluated in ESCC PDX tissues by immunohistochemistry. The immunohistochemical analysis results showed that the expression of Ki67 and p-ERK1/2 were strongly suppressed in the dasabuvir treatment group compared with the vehicle group (Fig. $6 e$ and $6 f$ ). These results indicate that dasabuvir can inhibit ESCC in vivo.

\section{Discussion}

The high recurrence rate and poor survival after treatment in patients with ESCC make the ongoing investigation of ESCC chemoprevention particularly important. FDA-approved drugs have detailed pharmacokinetic and safety data, making them a good choice for finding chemopreventive drugs for cancer. Through screening FDA-approved clinical drugs (Fig. 1a and Supplement Fig. S1a), we found dasabuvir, the anti-hepatitis $\mathrm{C}$ virus drug had an inhibitory effect on ESCC.

Dasabuvir had significant cytotoxic effects on ESCC cell lines KYSE150 and KYSE450 and significantly inhibited the growth and clonal formation of KYSE150 and KYSE450. Dasabuvir also inhibited the growth of PDX-inhibited ESCC in vivo. Most importantly, dasabuvir inhibited the progression of ESCC by inhibiting the ROCK1/ERK signaling pathway, which is different from previous studies showing that dasabuvir inhibits non-nucleoside NS5B polymerase in the treatment of hepatitis $\mathrm{C}$.

ROCK1 was reported to be involved in the progression of several cancers, including ESCC[23-25]. In addition, increased ROCK1 mRNA or ROCK1 protein expression can lead to a negative impact on the survival of patients, and a positive effect on the progression and prognosis of the disease[37]. Currently, various ROCK1 inhibitors are used to reduce progression, metastasis and migration of a variety of cancers[37]. Hundreds of ROCK1 inhibitors have been 
identified and have various therapeutic potentials. ROCK1/2 inhibitors such as fasudil[38, 39], AT13148[40-42], Y-27632[43], YM529/ONO-5920[44, 45], PT262[46], WF-536[47-49] and RKI-1447[50] have shown significant cancer inhibition[11].

Current reports on the relationship between ROCK1 and ERK are inconsistent. For example, ROCK regulated the upstream of ERK[30, 51], ROCK1 directly regulated ERK independently of MEK1/2[52], ROCK1 negatively regulated ERK activity[52], ROCK1 promoted the phosphorylation of ERK[31, 53]. In this study, we found ROCK1 could directly phosphorylate ERK, then dasabuvir inhibited the phosphorylation of ERK by ROCK1(Fig. 3J and 3k). This finding provides new scientific evidence for the study of the relationship between ROCK1 and ERK.

In addition, ROCK1 played an important role in the regulation of cell cycle progression. ROCK promoted cell cycle transition from $\mathrm{G} 1$ phase to $\mathrm{S}$ phase by upregulating cyclin A/D1/D3, CDK2/4/6 and downregulating cell cycle inhibitors CDKN1A, CDKN1B, CDKN2A, CDKN2C, CDKN2D and CDKN4B[54]. ROCK activated the RAS /MAPK pathway and increased the expression of cyclin D1[55]. The results of GSEA enrichment in this study demonstrated that dasabuvir did affect the cell cycle signaling pathway in ESCC(Fig. 4b and 4c), proteomics(Fig. 4d-4f), Western blotting(Fig. 4g) and cell cycle(Fig. 4h) results also showed that dasabuvir suppressed the expression of CDK4 and cyclin D1, thus blocking the cells in the G0/G1 phase.

As a whole, the results of the study suggest that dasabuvir bound to ROCK1 at Met156, Leu202, and Asp205. Dasabuvir acted on ROCK1, and inhibited the phosphorylation of ERK1/2, downregulating the expression of CDK4 and cyclin D1, which arrested cells in the G0/G1 phase of the cell cycle. Knockdown of ROCK1 in ESCC cells effectively inhibited cell proliferation and weakened the inhibitory effect of dasabuvir (Fig. 7).

\section{Conclusion}

This study found a novel effect of dasabuvir on cancer. As a ROCK1 inhibitor, dasabuvir inhibited the phosphorylation of ERK1/2 and downregulated CDK4 and cyclin D1, thus blocking the progression of ESCC. These results could be benefit for both the future research and clinical use of dasabuvir.

\section{Abbreviations}

ESCC, Esophageal squamous cell carcinoma; ROCK1, Rho-associated protein kinase1; ERK, extracellular signal-regulated kinase 1/2; CDK4, Cyclin-dependent kinase 4; PDX, Patient-derived Xenograft

\section{Declarations}

\section{Acknowledgements}

Not applicable.

\section{Authors' contributions}

$\mathrm{XNL}, \mathrm{KDL}$, and YNJ designed the experiments and wrote the manuscript; XNL performed cell phenotypes, immunofluorescence, Western blotting, animal experiments; HZ. and JMZ collected and processed data; MZL and ZPG prepared the reagents; ZB and ZGD performed immunohistochemical staining; ZTW constructed shROCK1 cells; CYZ and ZLX purified the ROCK1 protein; YNJ and KDL reviewed and revised the manuscript. All authors read and approved the final manuscript.

\section{Funding}

This work was supported by the National Natural Science Foundations of China (grant number 81872335), National Natural Science Youth Foundation (grant number 81902486), the Natural Science Foundation of Henan (grant number 161100510300), National Science \& Technology Major Project Key New Drug Creation and Manufacturing Program, China (grant number 2018ZX09711002), the Science and Technology Project of Henan Province (grant number 212102310187).

\section{Availability of data and materials}

The data about this study are available from the corresponding author on reasonable request.

\section{Ethics approval and consent to participate}

All animal experiments were approved by the Zhengzhou University Ethics

Committee (Zhengzhou, Henan, China). All of the cancer tissues used in this study were approved by the cancer patients.

\section{Consent for publication}

All authors agree with the manuscript.

\section{Competing interests}

The authors declare no competing interests.

\section{Author details}


${ }^{1}$ Department of Pathophysiology, School of Basic Medical Sciences, Zhengzhou University, Zhengzhou, Henan, China. ${ }^{2}$ China-US Hormel (Henan) Cancer Institute, Zhengzhou, Henan, China. ${ }^{3}$ State Key Laboratory of Esophageal Cancer Prevention and Treatment, Zhengzhou University, Zhengzhou, 450052 China. ${ }^{4}$ Henan Provincial Cooperative Innovation Center for Cancer Chemoprevention, Zhengzhou, China. ${ }^{5}$ Fuwai Central China Cardiovascular Hospital, Zhengzhou University, Zhengzhou, China. ${ }^{6}$ Cancer Chemoprevention International Collaboration Laboratory, Zhengzhou, China. ${ }^{7}$ Research Center of Basic Medicine区 Academy of Medical Sciences $₫$ Zhengzhou University.

\section{References}

1. Sung H, Ferlay J, Siegel RL, Laversanne M, Soerjomataram I, Jemal A, Bray F. Global cancer statistics 2020: GLOBOCAN estimates of incidence and mortality worldwide for 36 cancers in 185 countries. CA Cancer J Clin 2021.

2. Codipilly DC, Qin Y, Dawsey SM, Kisiel J, Topazian M, Ahlquist D, lyer PG. Screening for esophageal squamous cell carcinoma: recent advances. GASTROINTEST ENDOSC. 2018;88(3):413-26.

3. Harada K, Rogers JE, Iwatsuki M, Yamashita K, Baba H, Ajani JA: Recent advances in treating oesophageal cancer. F1000Res $2020,9$.

4. Yang J, Liu X, Cao S, Dong X, Rao S, Cai K. Understanding Esophageal Cancer: The Challenges and Opportunities for the Next Decade. FRONT ONCOL. 2020;10:1727

5. Yuan Q, Dong CD, Ge Y, Chen X, Li Z, Li X, Lu Q, Peng F, Wu X, Zhao J, et al. Proteome and phosphoproteome reveal mechanisms of action of atorvastatin against esophageal squamous cell carcinoma. Aging. 2019;11(21):9530-43.

6. Mohs RC, Greig NH. Drug discovery and development: Role of basic biological research. Alzheimers Dement (N Y). 2017;3(4):651-7.

7. Prasad V, Mailankody S. Research and Development Spending to Bring a Single Cancer Drug to Market and Revenues After Approval. JAMA INTERN MED. 2017;177(11):1569-75.

8. Kohli A, Alshati A, Georgie F, Manch R, Gish RG. Direct-acting antivirals for the treatment of chronic hepatitis C in patients with chronic kidney disease. Therap Adv Gastroenterol. 2016;9(6):887-97.

9. Suwanthawornkul T, Anothaisintawee T, Sobhonslidsuk A, Thakkinstian A, Teerawattananon Y: Efficacy of Second Generation Direct-Acting Antiviral Agents for Treatment Naive Hepatitis C Genotype 1: A Systematic Review and Network Meta-Analysis. PLOS ONE 2015, 10(12):e145953.

10. Mantry PS, Pathak L. Dasabuvir (ABT333) for the treatment of chronic HCV genotype I: a new face of cure, an expert review. Expert Rev Anti Infect Ther. 2016;14(2):157-65.

11. de Sousa GR, Vieira GM, Das CP, Pezuk JA, Brassesco MS. Should we keep rocking? Portraits from targeting Rho kinases in cancer. PHARMACOL RES. 2020;160:105093.

12. Rath N, Olson MF. Rho-associated kinases in tumorigenesis: re-considering ROCK inhibition for cancer therapy. EMBO REP. 2012;13(10):900-8.

13. Liu K, Li X, Wang J, Wang Y, Dong H, Li J. Genetic variants in RhoA and ROCK1 genes are associated with the development, progression and prognosis of prostate cancer. Oncotarget. 2017;8(12):19298-309.

14. Yang J, Zhao S, Li B. Long noncoding RNA PANDAR promotes progression and predicts poor prognosis via upregulating ROCK1 in prostate cancer. Eur Rev Med Pharmacol Sci. 2019;23(11):4706-12.

15. Gong H, Zhou L, Khelfat L, Qiu G, Wang Y, Mao K, Chen W. Rho-Associated Protein Kinase (ROCK) Promotes Proliferation and Migration of PC-3 and DU145 Prostate Cancer Cells by Targeting LIM Kinase 1 (LIMK1) and Matrix Metalloproteinase-2 (MMP-2). Med Sci Monit. 2019;25:3090-9.

16. Steurer S, Hager B, Buscheck F, Hoflmayer D, Tsourlakis MC, Minner S, Clauditz TS, Hube-Magg C, Luebke AM, Simon R, et al. Up regulation of Rhoassociated coiled-coil containing kinase1 (ROCK1) is associated with genetic instability and poor prognosis in prostate cancer. Aging. 2019;11(18):785979 .

17. Zhang J, He X, Ma Y, Liu Y, Shi H, Guo W, Liu L. Overexpression of ROCK1 and ROCK2 inhibits human laryngeal squamous cell carcinoma. Int J Clin Exp Pathol. 2015;8(1):244-51.

18. Wang X, Huang Y, Guo R, Liu Y, Qian Y, Liu D, Dai X, Wei Z, Jin F, Liu Y. Clinicopathological significance of ROCK1 and PIK3CA expression in nasopharyngeal carcinoma. EXP THER MED. 2017;13(3):1064-8.

19. Shi D, Wu F, Mu S, Hu B, Zhong B, Gao F, Qing X, Liu J, Zhang Z, Shao Z. LncRNA AFAP1-AS1 promotes tumorigenesis and epithelial-mesenchymal transition of osteosarcoma through RhoC/ROCK1/p38MAPK/Twist1 signaling pathway. J Exp Clin Cancer Res. 2019;38(1):375.

20. Wang Y, Zeng X, Wang N, Zhao W, Zhang X, Teng S, Zhang Y, Lu Z. Long noncoding RNA DANCR, working as a competitive endogenous RNA, promotes ROCK1-mediated proliferation and metastasis via decoying of miR-335-5p and miR-1972 in osteosarcoma. MOL CANCER. 2018;17(1):89.

21. Gilkes DM, Xiang L, Lee SJ, Chaturvedi P, Hubbi ME, Wirtz D, Semenza GL. Hypoxia-inducible factors mediate coordinated RhoA-ROCK1 expression and signaling in breast cancer cells. Proc Natl Acad Sci U S A. 2014;111(3):E384-93.

22. Zheng B, Liang L, Wang C, Huang S, Cao X, Zha R, Liu L, Jia D, Tian Q, Wu J, et al. MicroRNA-148a suppresses tumor cell invasion and metastasis by downregulating ROCK1 in gastric cancer. CLIN CANCER RES. 2011;17(24):7574-83.

23. Zhu P, Yu H, Zhou K, Bai Y, Qi R, Zhang S. 3,3'-Diindolylmethane modulates aryl hydrocarbon receptor of esophageal squamous cell carcinoma to reverse epithelial-mesenchymal transition through repressing RhoA/ROCK1-mediated COX2/PGE2 pathway. J Exp Clin Cancer Res. 2020;39(1):113.

24. Zhou J, Zhao LQ, Xiong MM, Wang XQ, Yang GR, Qiu ZL, Wu M, Liu ZH. Gene expression profiles at different stages of human esophageal squamous cell carcinoma. World J Gastroenterol. 2003;9(1):9-15.

25. Sasaki Y, Tamura M, Koyama R, Nakagaki T, Adachi Y, Tokino T. Genomic characterization of esophageal squamous cell carcinoma: Insights from nextgeneration sequencing. World J Gastroenterol. 2016;22(7):2284-93.

Page $8 / 16$ 
26. Zhao S, Jiang Y, Zhao J, Li H, Yin X, Wang Y, Xie Y, Chen X, Lu J, Dong Z, et al. Quercetin-3-methyl ether inhibits esophageal carcinogenesis by targeting the AKT/mTOR/p70S6K and MAPK pathways. Mol Carcinog. 2018;57(11):1540-52.

27. Zhao R, Choi BY, Wei L, Fredimoses M, Yin F, Fu X, Chen H, Liu K, Kundu JK, Dong Z, et al. Acetylshikonin suppressed growth of colorectal tumour tissue and cells by inhibiting the intracellular kinase, T-lymphokine-activated killer cell-originated protein kinase. Br J Pharmacol. 2020;177(10):2303-19.

28. Tian X, Liu K, Zu X, Ma F, Li Z, Lee M, Chen H, Li Y, Zhao Y, Liu F, et al: 3,3'-Diindolylmethane inhibits patient-derived xenograft colon tumor growth by targeting COX1/2 and ERK1/2. CANCER LETT 2019, 448:20-30.

29. Guo YJ, Pan WW, Liu SB, Shen ZF, Xu Y, Hu LL. ERK/MAPK signalling pathway and tumorigenesis. EXP THER MED. 2020;19(3):1997-2007.

30. Wei YH, Liao SL, Wang SH, Wang CC, Yang CH. Simvastatin and ROCK Inhibitor Y-27632 Inhibit Myofibroblast Differentiation of Graves' OphthalmopathyDerived Orbital Fibroblasts via RhoA-Mediated ERK and p38 Signaling Pathways. Front Endocrinol (Lausanne). 2020;11:607968.

31. Zhao Y, Lv M, Lin H, Hong Y, Yang F, Sun Y, Guo Y, Cui Y, Li S, Gao Y. ROCK1 induces ERK nuclear translocation in PDGF-BB-stimulated migration of rat vascular smooth muscle cells. IUBMB LIFE. 2012;64(2):194-202.

32. Zhao Y, Lv M, Lin H, Cui Y, Wei X, Qin Y, Kohama K, Gao Y. Rho-associated protein kinase isoforms stimulate proliferation of vascular smooth muscle cells through ERK and induction of cyclin D1 and PCNA. Biochem Biophys Res Commun. 2013;432(3):488-93.

33. Jacobs M, Hayakawa K, Swenson L, Bellon S, Fleming M, Taslimi P, Doran J. The structure of dimeric ROCK I reveals the mechanism for ligand selectivity. J BIOL CHEM. 2006;281(1):260-8.

34. Tang L, Dai F, Liu Y, Yu X, Huang C, Wang Y, Yao W. RhoA/ROCK signaling regulates smooth muscle phenotypic modulation and vascular remodeling via the JNK pathway and vimentin cytoskeleton. PHARMACOL RES. 2018;133:201-12.

35. Jung J, Seol HS, Chang S. The Generation and Application of Patient-Derived Xenograft Model for Cancer Research. CANCER RES TREAT. 2018;50(1):110.

36. Jiang Y, Wu Q, Yang X, Zhao J, Jin Y, Li K, Ma Y, Chen X, Tian F, Zhao S, et al. A method for establishing a patient-derived xenograft model to explore new therapeutic strategies for esophageal squamous cell carcinoma. ONCOL REP. 2016;35(2):785-92.

37. Shahbazi R, Baradaran B, Khordadmehr M, Safaei S, Baghbanzadeh A, Jigari F, Ezzati H. Targeting RocK signaling in health, malignant and nonmalignant diseases. IMMUNOL LETT. 2020;219:15-26.

38. Huo Z, Su Y, Dong Y, Zheng Y, Zhang Q, Duan Y, Wang G. Rho-kinase inhibition by Fasudil promotes tumor maturation and apoptosis in small-cell lung cancer. AM J TRANSL RES. 2020;12(8):4354-70.

39. Zhao Y, Zhang Y, Vazirinejad MM, Zhou K, Chen Y, Li L, Guo J, Xu C. Enhanced anti-tumor effect of liposomal Fasudil on hepatocellular carcinoma in vitro and in vivo. PLOS ONE. 2019;14(10):e223232.

40. Sadok A, McCarthy A, Caldwell J, Collins I, Garrett MD, Yeo M, Hooper S, Sahai E, Kuemper S, Mardakheh FK, et al. Rho kinase inhibitors block melanoma cell migration and inhibit metastasis. CANCER RES. 2015;75(11):2272-84.

41. Yap TA, Walton MI, Grimshaw KM, Te PR, Eve PD, Valenti MR, de Haven BA, Martins V, Zetterlund A, Heaton SP, et al. AT13148 is a novel, oral multi-AGC kinase inhibitor with potent pharmacodynamic and antitumor activity. CLIN CANCER RES. 2012;18(14):3912-23.

42. Rath N, Munro J, Cutiongco MF, Jagiello A, Gadegaard N, McGarry L, Unbekandt M, Michalopoulou E, Kamphorst JJ, Sumpton D, et al. Rho Kinase Inhibition by AT13148 Blocks Pancreatic Ductal Adenocarcinoma Invasion and Tumor Growth. CANCER RES. 2018;78(12):3321-36.

43. Zhu Y, Howard GA, Pittman K, Boykin C, Herring LE, Wilkerson EM, Verbanac K, Lu Q. Therapeutic Effect of Y-27632 on Tumorigenesis and CisplatinInduced Peripheral Sensory Loss through RhoA-NF-kappaB. MOL CANCER RES. 2019;17(9):1910-9.

44. Tsubaki M, Satou T, Itoh T, Imano M, Ogaki M, Yanae M, Nishida S. Reduction of metastasis, cell invasion, and adhesion in mouse osteosarcoma by YM529/ONO-5920-induced blockade of the Ras/MEK/ERK and Ras/PI3K/Akt pathway. Toxicol Appl Pharmacol. 2012;259(3):402-10.

45. Tanimori Y, Tsubaki M, Yamazoe Y, Satou T, Itoh T, Kidera Y, Yanae M, Yamamoto C, Kaneko J, Nishida S. Nitrogen-containing bisphosphonate, YM529/ONO-5920, inhibits tumor metastasis in mouse melanoma through suppression of the Rho/ROCK pathway. Clin Exp Metastasis. 2010;27(7):52938.

46. Hsu TS, Chen C, Lee PT, Chiu SJ, Liu HF, Tsai CC, Chao JI: 7-Chloro-6-piperidin-1-yl-quinoline-5,8-dione (PT-262), a novel synthetic compound induces lung carcinoma cell death associated with inhibiting ERK and CDC2 phosphorylation via a p53-independent pathway. Cancer Chemother Pharmaco/2008, 62(5):799-808.

47. Nakajima M, Katayama K, Tamechika I, Hayashi K, Amano Y, Uehata M, Goto N, Kondo T. WF-536 inhibits metastatic invasion by enhancing the host cell barrier and inhibiting tumour cell motility. Clin Exp Pharmacol Physiol. 2003;30(7):457-63.

48. Nakajima M, Hayashi K, Katayama K, Amano Y, Egi Y, Uehata M, Goto N, Kondo T. Wf-536 prevents tumor metastasis by inhibiting both tumor motility and angiogenic actions. EUR J PHARMACOL. 2003;459(2-3):113-20.

49. Nakajima M, Hayashi K, Egi Y, Katayama K, Amano Y, Uehata M, Ohtsuki M, Fujii A, Oshita K, Kataoka H, et al. Effect of Wf-536, a novel ROCK inhibitor, against metastasis of B16 melanoma. Cancer Chemother Pharmacol. 2003;52(4):319-24.

50. Li L, Chen Q, Yu Y, Chen H, Lu M, Huang Y, Li P, Chang H. RKI-1447 suppresses colorectal carcinoma cell growth via disrupting cellular bioenergetics and mitochondrial dynamics. J CELL PHYSIOL. 2020;235(1):254-66.

51. Ung CY, Li H, Ma XH, Jia J, Li BW, Low BC, Chen YZ. Simulation of the regulation of EGFR endocytosis and EGFR-ERK signaling by endophilin-mediated RhoA-EGFR crosstalk. FEBS LETT. 2008;582(15):2283-90.

52. Li F, Jiang Q, Shi KJ, Luo H, Yang Y, Xu CM. RhoA modulates functional and physical interaction between ROCK1 and Erk1/2 in selenite-induced apoptosis of leukaemia cells. CELL DEATH DIS. 2013;4:e708. 
53. Chaturvedi LS, Marsh HM, Basson MD. Role of RhoA and its effectors ROCK and mDia1 in the modulation of deformation-induced FAK, ERK, p38, and MLC motogenic signals in human Caco-2 intestinal epithelial cells. Am J Physiol Cell Physiol. 2011;301(5):C1224-38.

54. Street CA, Bryan BA. Rho kinase proteins-pleiotropic modulators of cell survival and apoptosis. ANTICANCER RES. 2011;31(11):3645-57.

55. Croft DR, Olson MF. The Rho GTPase effector ROCK regulates cyclin A, cyclin D1, and p27Kip1 levels by distinct mechanisms. MOL CELL BIOL. 2006;26(12):4612-27.

\section{Figures}

a

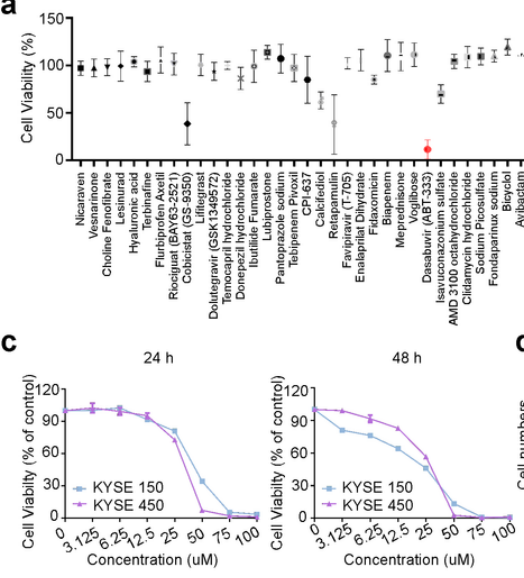

e

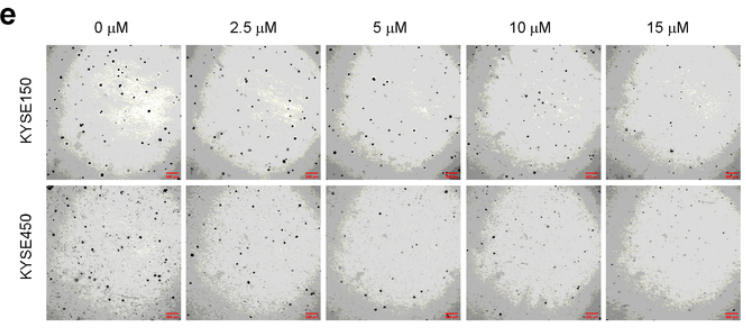

$\mathbf{f}$
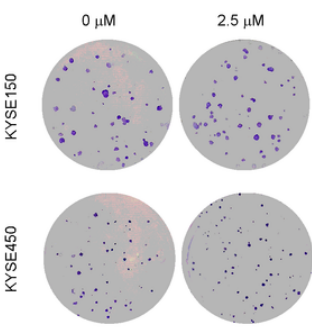
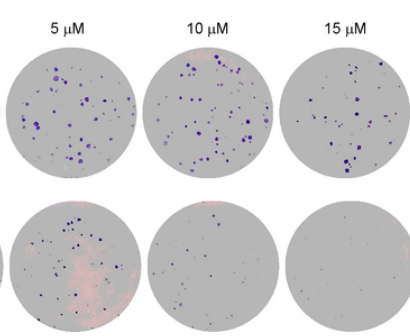

b

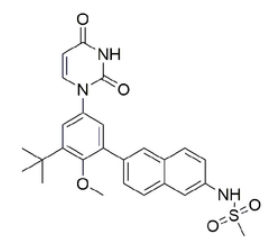

KYSE450

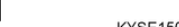

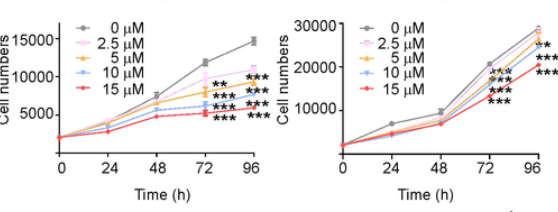
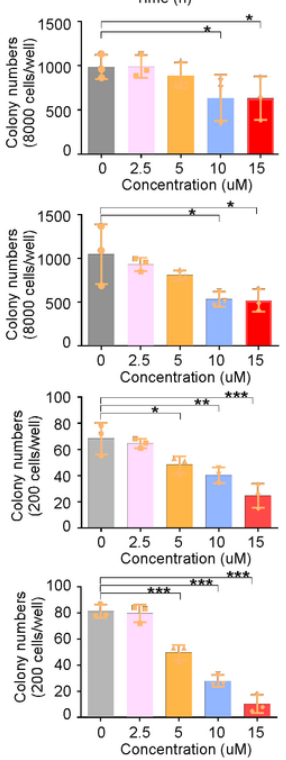

Figure 1

Dasabuvir inhibits ESCC proliferation in vitro. (A) KYSE450 cells were used to screen for cytotoxicity of 50 kinds of drugs ( $n=3$ ). (B) Chemical structure of dasabuvir. (C) The effect of dasabuvir on the cell viability of KYSE150, KYSE450 cells. Cell viability was detected by DAPI staining ( $n=3$ ). (D) The effect of dasabuvir on the proliferation of KYSE150, KYSE450 cells. Proliferation ability was detected by DAPI staining $(n=3)$. (E) Representative images of anchorageindependent cell growth assay (left) and quantitative analyses of colony numbers (right, $n=3$ ). (F) Representative images of anchorage-dependent cell growth assay (left) and quantitative analyses of colony numbers (right, $n=3$ ). Data were analyzed by one-way ANOVA test and the asterisk indicated a significant $\left({ }^{*} \mathrm{p}\right.$ $<0.05, * * p<0.01$ and $\left.{ }^{* \star *} p<0.001\right)$ difference compared with the control group. 


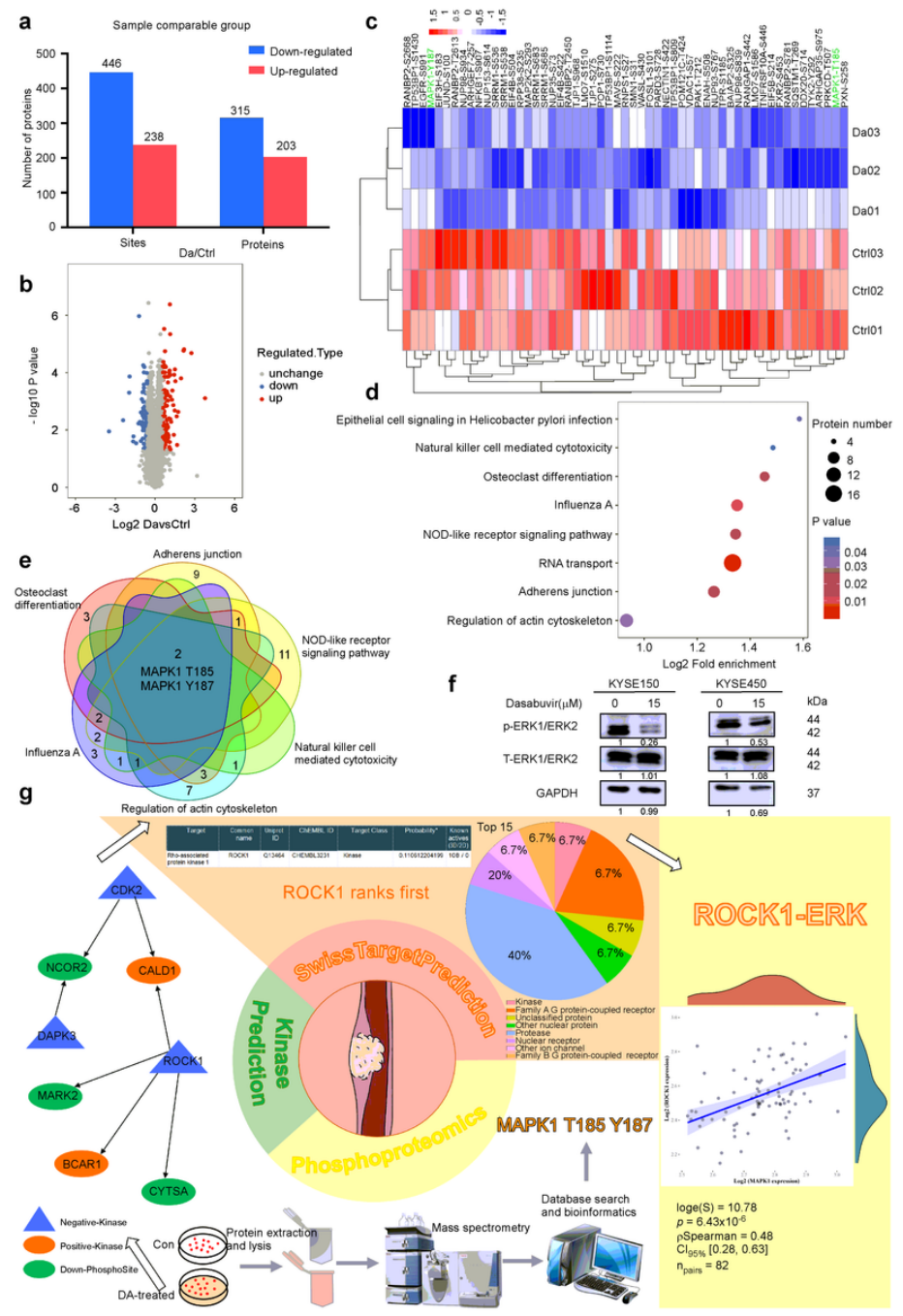

\section{Figure 2}

Omics data indicated that Dasabuvir played the role via ROCK1/MAPK1 signal pathway (A) Histogram showing the number of changed expressed protein and phosphorylated protein site between control and dasabuvir $(15 \mu \mathrm{M})$ treated group in KYSE150 cells in phosphoproteomics. (B) Volcano diagram showing the most important changed phosphorylated protein site between control and dasabuvir (15 $\mu \mathrm{M}$ ) treated group in KYSE150 cells. (C) Heatmap showing significantly down-regulated phosphorylated protein sites in KYSE150. (D) The enrichment KEGG pathway analysis of the downregulated expression proteins on phosphorylated level. (E) MAPK1 T185, Y187 were enriched from the KEGG pathways. (F) The selected target was validated by Western blotting. Representative images are shown. (G) The flow chart showing how we found the ROCK1/ERK signaling pathway. 

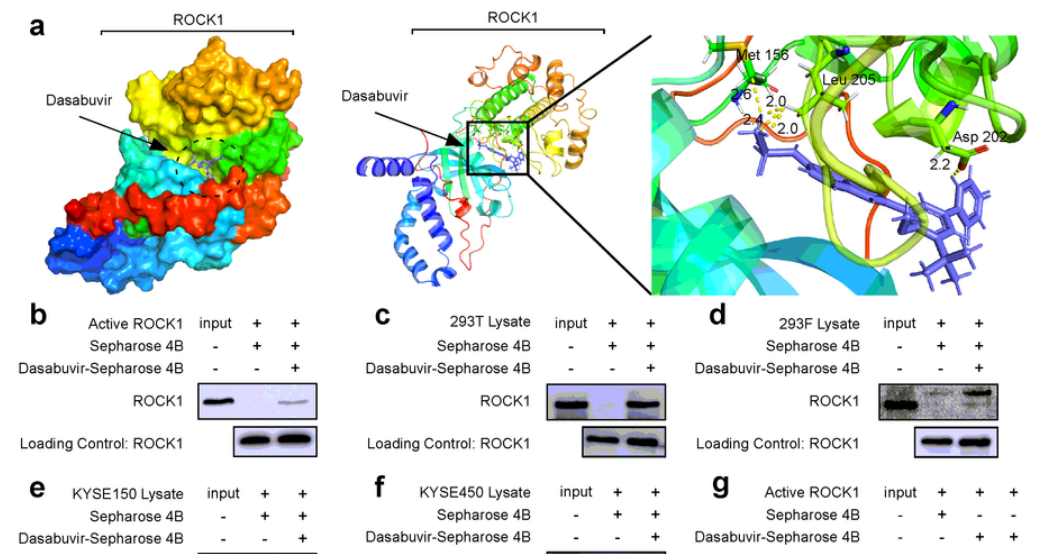

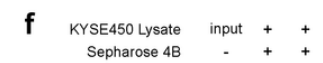
Dasabuvir-Sepharose 4B - . + ROCK1

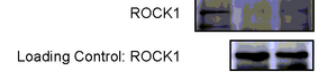
Loading Control: ROCK1

$E=$
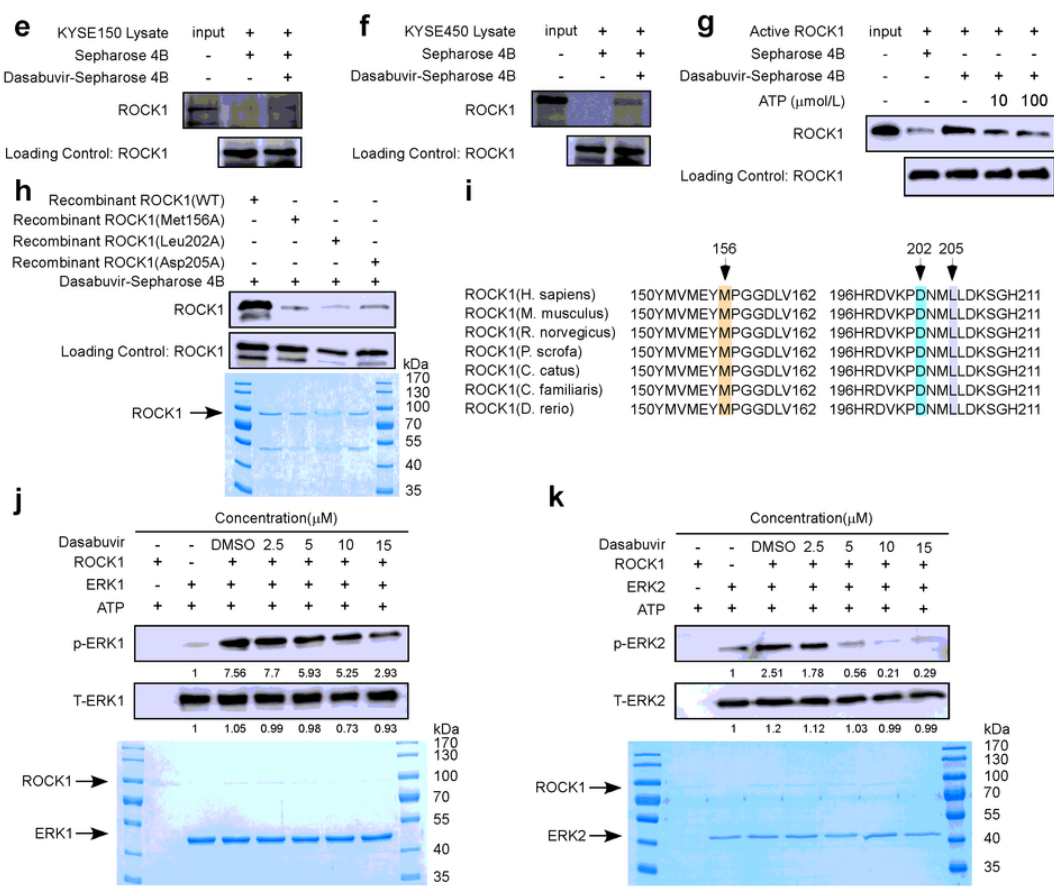

\section{Figure 3}

Dasabuvir binds and inhibits ROCK1 (A) Computer molecular simulation showing dasabuvir could bind with ROCK1 kinase domain (PBD: 2ESM). (B) In vitro pulldown assay indicated dasabuvir could bind to recombinant ROCK1 protein. (C and D) Ex vivo pulldown assay indicated dasabuvir could bind to the fulllength ROCK1. (E and F) In vivo pulldown indicated dasabuvir could bind to endogenous ROCK1. (G) ATP competition assay indicated dasabuvir bound to ROCK1 in an ATP an ATP competitive way. (H) Dasabuvir bound with WT ROCK1 not mutant ROCK1 (156, 202, 205) validated by the pull down assay (upper). The SDS-PAGE gel showing the presence of wild type and mutant type ROCK1 proteins (lower). (I) Comparison diagram of ROCK1 sequence of different species. (J) In vitro kinase experiment assay showed ROCK1 can activate ERK1, while dasabuvir can inhibit this activation(upper). The SDS-PAGE gel showing the presence of ROCK1 and ERK1 (lower). (K) In vitro kinase experiment assay showed ROCK1 can activate ERK2, while dasabuvir can inhibit this activation(upper). The SDS-PAGE gel showing the presence of ROCK1 and ERK2 (lower). 
a
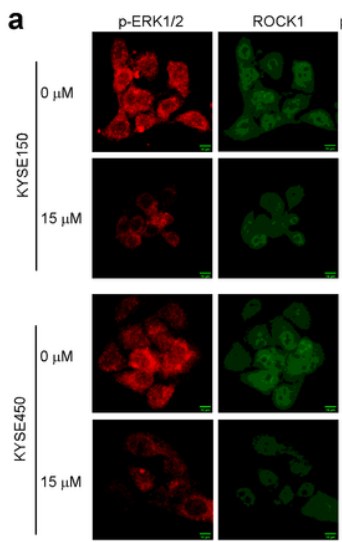

d

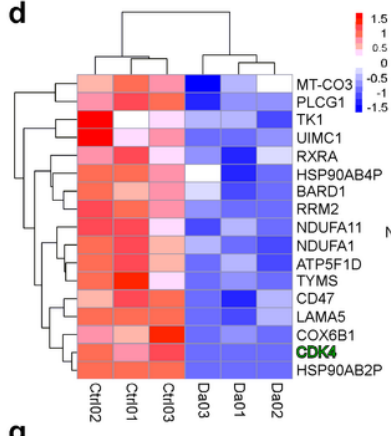

g
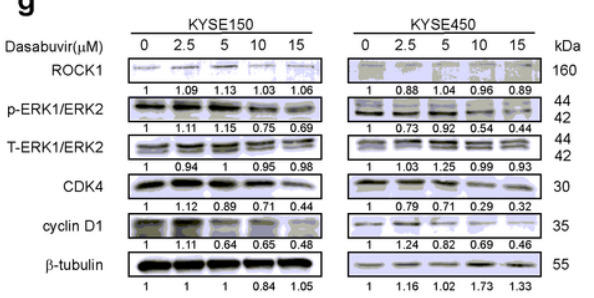

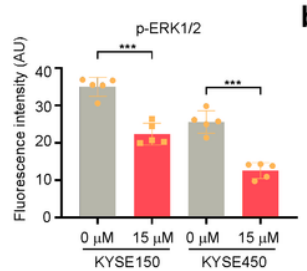

b
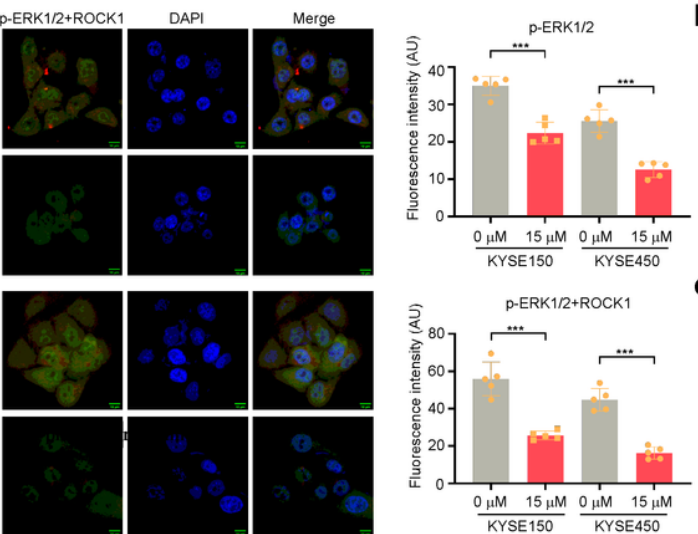

c

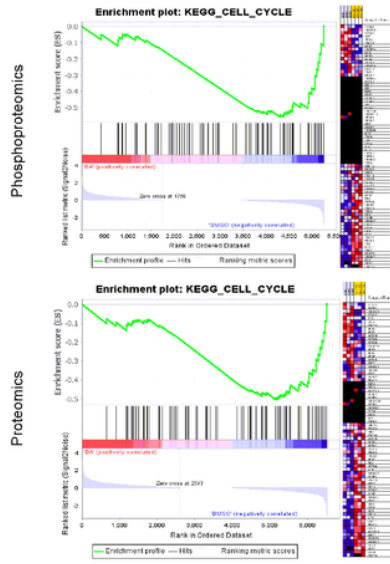

f
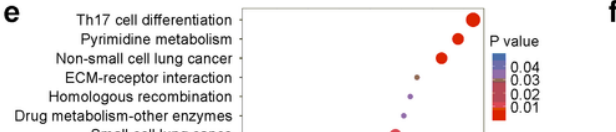

Small cell lung cance

IL-17 signaling pathway

Cardiac muscle contraction

Parkinson disease
Oxidative phosphorlation

Alzheimer disease

kt signaling pathway

Huntington disease

Thermogenesis .

Pathways in cancer

h
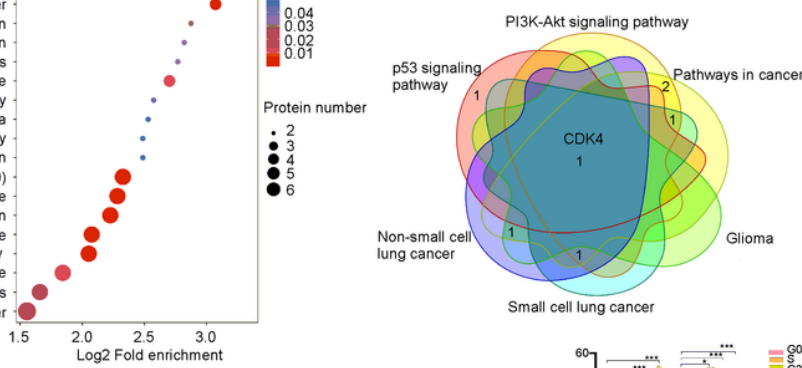

Small cell lung cancer
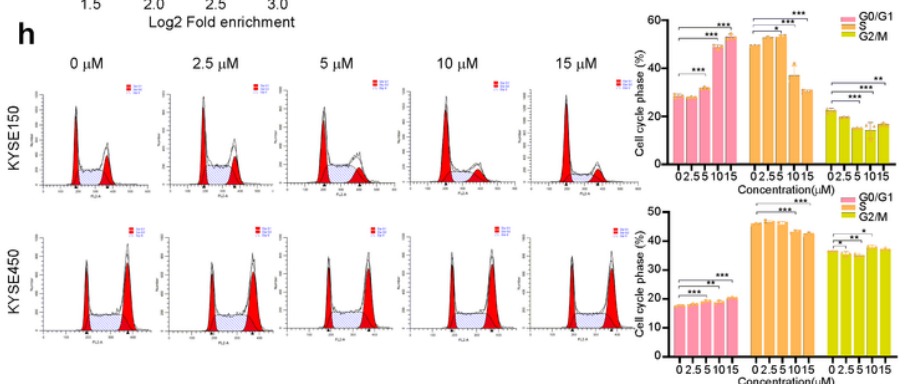

Figure 4

Dasabuvir induced G0-G1 cell cycle arrest of KYSE150 and KYSE450 cells by ROCK1/ERK signal pathway (A) Immunofluorescence of p-ERK1/2 and ROCK1 in KYSE150 and KYSE450 cells treated with dasabuvir $(15 \mu \mathrm{M})$ for $24 \mathrm{~h}$ was photoed by scanning confocal microscopy. Representative images (left) and quantitative analyses of fluorescence intensity (right, $n=3$ ). (B) GSEA Enrichment plot (score curves) from phosphoproteomics. (C) GSEA Enrichment plot (score curves) from proteomics. (D) Heat map showing significantly down-regulated protein sites in KYSE150. (E) The enrichment KEGG pathway analysis of the downregulated proteins. (F) CDK4 was enriched from the KEGG pathways. (G) Western blotting showed dasabuvir inhibited the expressions of p-ERK1/2, CDK4 and Cyclin D1 in a concentration gradient. $(\mathrm{H})$ Cell cycle was analyzed by PI staining and the number of cells in each phase was analyzed by Modfit $(n=$ 3). Data were analyzed by one-way ANOVA test and the asterisk indicated a significant $\left({ }^{\star} p<0.05,{ }^{\star *} p<0.01\right.$ and $\left.{ }^{* \star *} p<0.001\right)$ difference compared with the control group. 

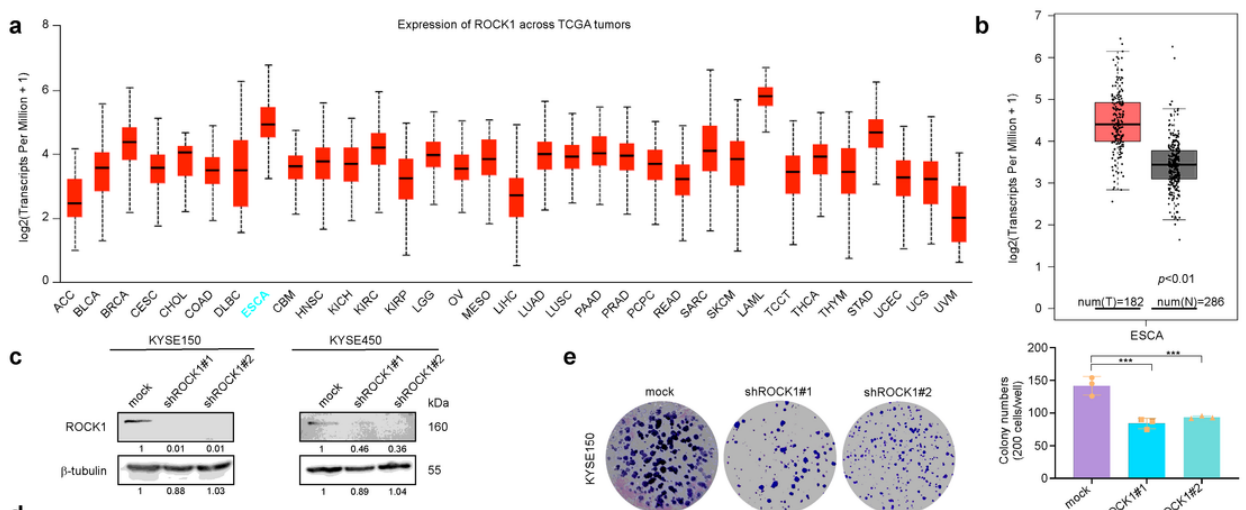

d KYSE 150
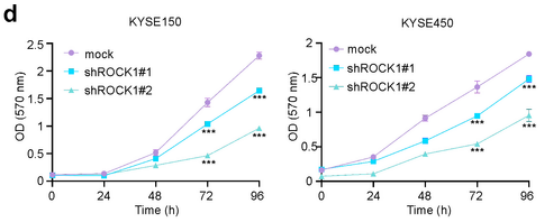

e
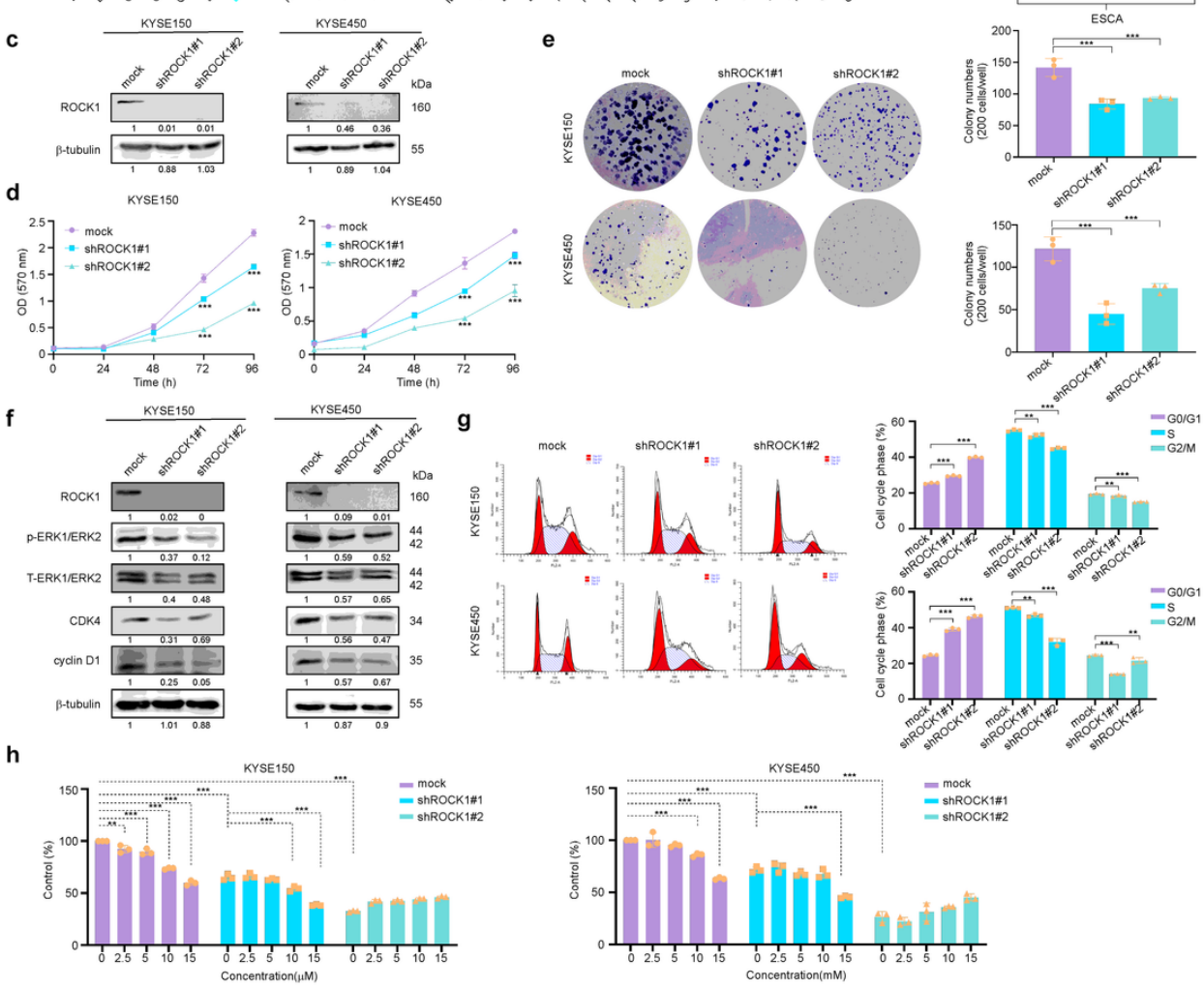

Figure 5

Dasabuvir exerts cancer cell inhibition through ROCK1 (A) The expression of ROCK1 across TCGA tumors from UALCAN. (B) The expression of ROCK1 between ESCA and normal tissues from GEPIA. (C) The expression of ROCK1 in KYSE150 and KYSE450 cells transfected with sh-Mock or shRNA-ROCK1 was measured by Western blotting. (D) After knocking down ROCK1, ESCC cell growth was estimated by MTT assay ( $n=3)$. (E) Representative images of anchorage-dependent cell growth assay (left) after ROCK1 knocking down and quantitative analyses of colony numbers (right, $n=3$ ). (F) The expressions of the corresponding downstream targets of after ROCK1 knocking down in KYSE150 and KYSE450 cells were measured by western blotting. (G) Cell cycle after knocking down ROCK1 was PI staining and the number of cells in each phase was analyzed by Modfit $(n=3)$. (H) The inhibitory effect of dasabuvir on shROCK1 cells was detected by MTT assay on $72 \mathrm{~h}(n=3)$. Data were analyzed by one-way ANOVA test and the asterisk indicated a significant $\left({ }^{*} p<0.05\right.$, ${ }^{* \star} p$ $<0.01$ and $\left.{ }^{* * *} \mathrm{p}<0.001\right)$ difference compared with the control group. 

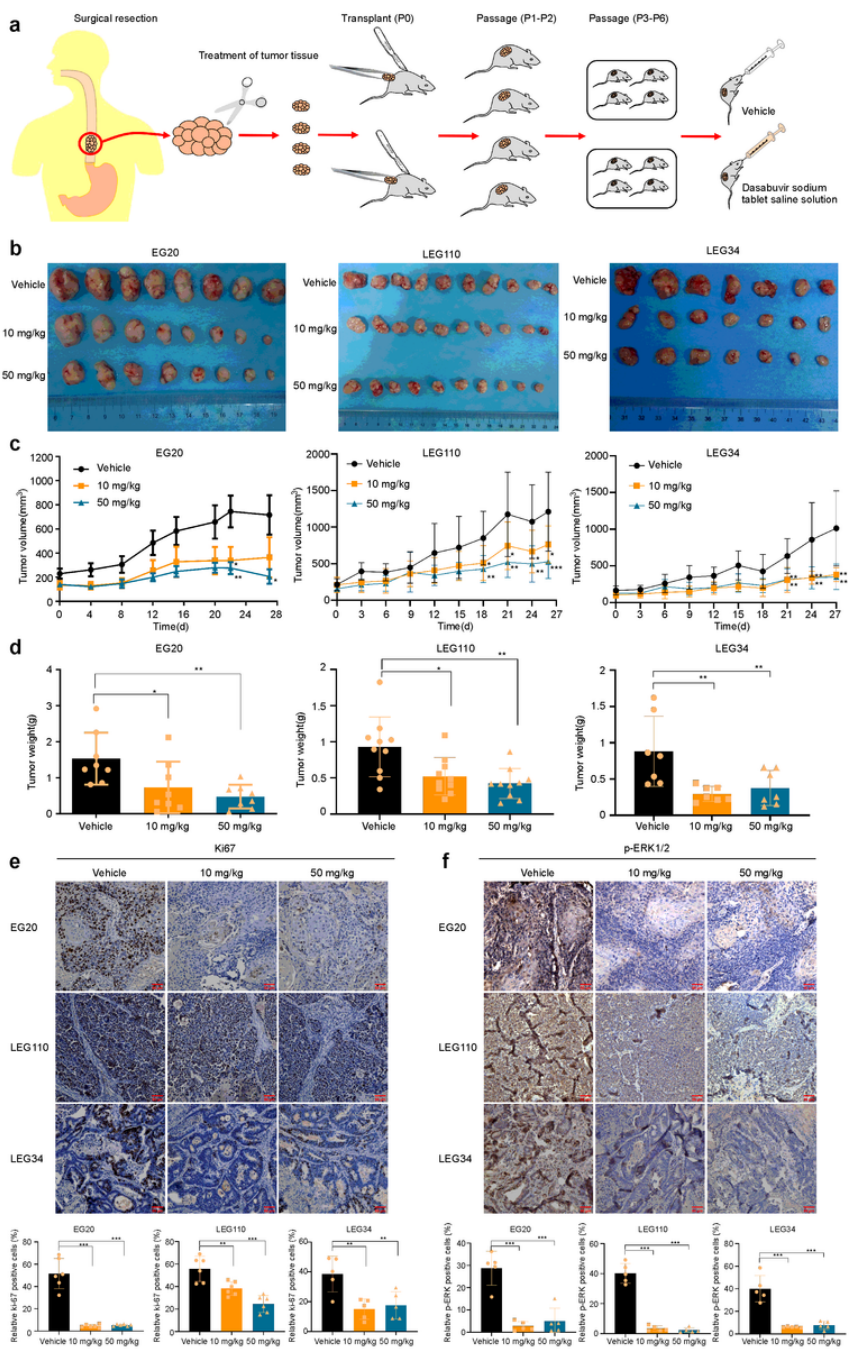

\section{Figure 6}

Dasabuvir reduced ESCC PDX xenograft growth in vivo Schematic diagram showing the process of PDX model construction. (B) Photographs of tumors for EG20 ( $n=8)$, LEG110 ( $n=10)$, LEG34 ( $n=7)$. (C) Tumor growth curve after dasabuvir treated for EG20 $(n=8)$, LEG110 $(n=10)$, LEG34 ( $n=7)$. (D) Tumor weight after dasabuvir treated for EG20 (n=8), LEG110 $(n=10)$, LEG34 $(n=7)$. (E) Ki67 expression levels in ESCC cancer PDX samples were examined by immunohistochemistry (IHC) for EG20 (n=6), LEG110 (n=5), LEG34 (n=6). (F) p-ERK1/2 expression levels were examined by IHC for EG20 ( $n=5)$, LEG110 (n=5), LEG34 $(n=5)$. Data were analyzed by one-way ANOVA test and the asterisk indicated a significant ( ${ }^{*}<0.05, * * p<0.01$ and $\left.* \star * p<0.001\right)$ difference compared with the control group. 


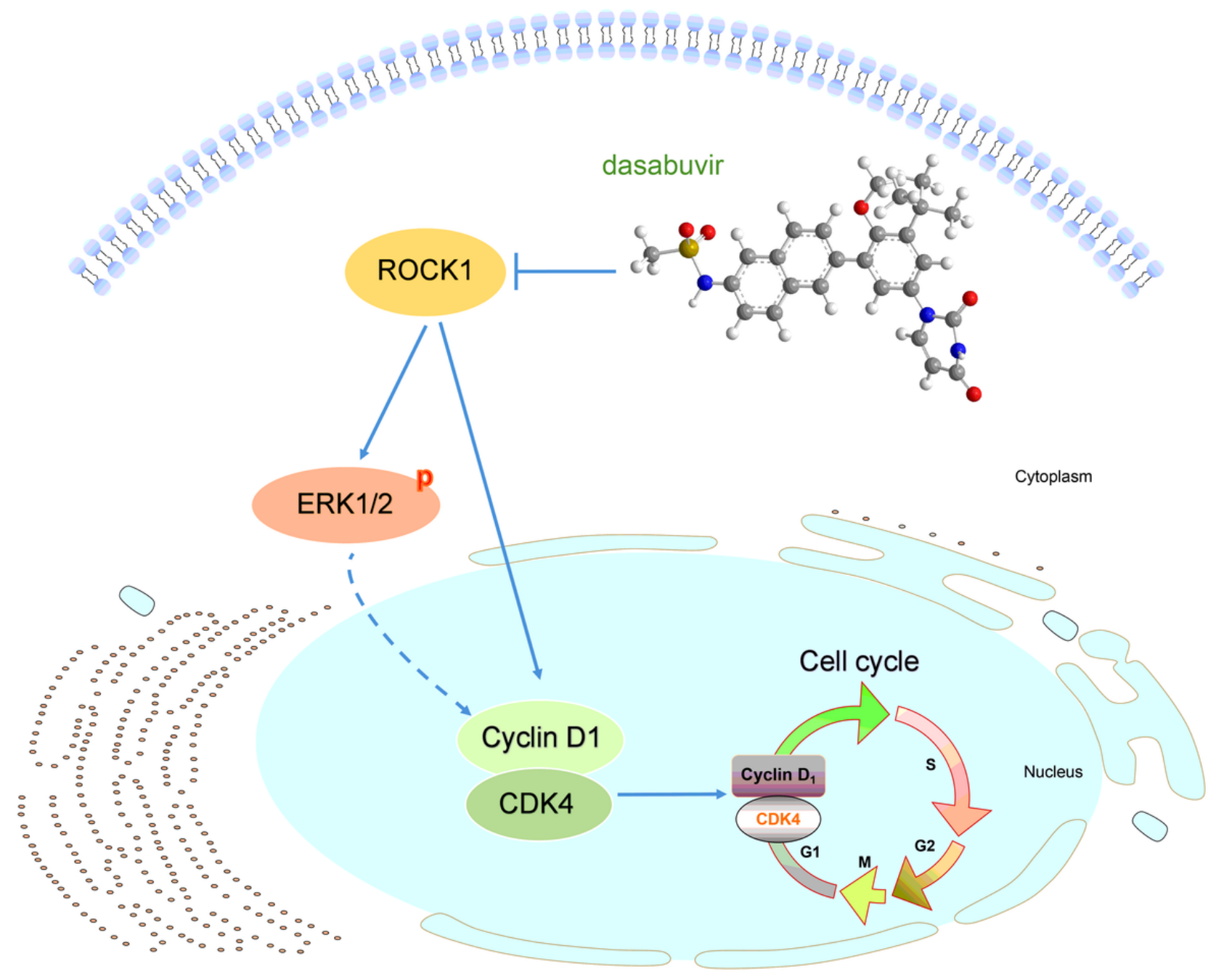

Figure 7

Schematic diagram showing the mechanism by dasabuvir inhibits esophageal squamous cell carcinoma

\section{Supplementary Files}

This is a list of supplementary files associated with this preprint. Click to download.
- su1.tif
- su2.tif
- su3.tif
- su4.tif 